\title{
Ophthalmology Clinic for the Homeless in San Francisco: Experiences and Findings
}

\author{
${ }^{1}$ School of Medicine, University of California San Francisco, San \\ Francisco, California \\ ${ }^{2}$ Department of Ophthalmology, University of California San \\ Francisco, San Francisco, California \\ J Acad Ophthalmol 2020;12:e214-e220.
}

Ogonna N. Nnamani Silva, MD ${ }^{1}$ Lauren Hennein, MD $^{2}$ Saras Ramanathan, MD²

Address for correspondence Saras Ramanathan, MD, Department of Ophthalmology, University of California, San Francisco, 400 Parnassus Avenue, San Francisco, CA 94143

(e-mail: Saras.Ramanathan@ucsf.edu).

\begin{abstract}
Keywords

- homelessness

- ophthalmology

- healthcare disparities

- student-run clinics

- ophthalmology education

- medical education
\end{abstract}

\begin{abstract}
Background Homelessness is a growing area of concern for communities around the world and homeless individuals are often not connected to healthcare and, consequently, this population is often left out of crucial studies that influence the care provided at public hospitals. The main aim of this study is to examine the prevalence of eye disease, demographics, and common comorbidities of patients who utilized the student-run, institution-sponsored ophthalmology clinic for homeless individuals across a 2-year period. A secondary aim was to elucidate the role that student-run clinics can play in connecting the homeless population to healthcare and community services.

Methods A retrospective cohort study examined individuals who utilized a studentrun, free ophthalmology clinic at two homeless shelters in an urban community for 2 years, from 2017 to 2019.

Results A volunteer sample consisting of 123 residents at two homeless shelters who signed up to receive an eye examination at the free eye clinic. The average age of participants was 51 years, with $74 \%$ identifying as male, and the largest number of patients identified as African-American/Black (33\%) and White (27\%). The most prevalent eye disease was refractive error at a prevalence rate of $70 \%$ with presbyopia and myopia accounting for $77 \%$ and $42 \%$ of the refractive error diagnoses, respectively. A visually significant cataract was the second most prevalent pathology, which was diagnosed in $13 \%$ of patients. Diabetic retinopathy had a prevalence rate of $11 \%$. Glaucoma had a prevalence rate of $20 \%$. When stratifying the prevalence of glaucoma by race/ethnicity, Black/African-Americans had a prevalence rate of $30 \%$ and Asians had a prevalence rate of $29 \%$.

Conclusion This study demonstrated a high prevalence of eye disease among the homeless population highlighting the major role that social determinants of health play in patient outcomes. Student-run ophthalmology clinics provide rich educational experiences, and most importantly, connect homeless populations to essential ophthalmologic care.
\end{abstract}

Homelessness is one of society's greatest challenges facing nearly 1 million in the United States each day ${ }^{1}$ and its prevalence has increased in communities across the United States. ${ }^{2}$ The effects of homelessness pose significant consequences at both the societal and individual level. At the societal level, homelessness costs the United States economy about \$2 trillion annually. ${ }^{3}$ At the individual level, strong associations exist between homelessness and a multitude of comorbidities received

June 2, 2020

accepted

August 31, 2020
DOI https://doi.org/

10.1055/s-0040-1718563. ISSN $2475-4757$.
Copyright $\odot 2020$ by Thieme Medical

Publishers, Inc., 333 Seventh Avenue, New York, NY 10001, USA. Tel: +1(212) 760-0888.
License terms

$\circledast(1) \Theta \circledast$ 
such as heart disease, diabetes, hypertension (HTN), depression, and substance use disorders. ${ }^{1,4-7}$ The homeless population faces higher barriers in accessing primary and preventive care services and as a result has higher rates of acute care utilization and hospitalizations. ${ }^{3,8}$ Despite homelessness being a widespread problem, there is limited information about the eye health of this population ${ }^{9,10}$ and the available resources for proper treatment. The current literature identifies a higher prevalence of eye disease among subsets of homeless populations located abroad, ${ }^{11-13}$ but multiyear comprehensive assessments of ophthalmologic health in this population within the United States are deficient. ${ }^{14}$ This study will be the first to investigate the ocular health of the homeless living in an urban setting in the United States.

With a rapidly growing homeless population and an anticipated increased need for ophthalmologic services, the University of California San Francisco Homeless Shelter Eye Clinic (UCSF-HSEC) was launched on August 23, 2017 at the Multi-Services South Homeless Shelter, and subsequently the Navigation Center in San Francisco.

The main aim of this study was to examine the prevalence of eye disease, demographics, and prevalence of comorbidities such as HTN, type 2 diabetes (DM2), and human immunodeficiency virus/acquired immunodeficiency syndrome (HIV/AIDS) of patients who utilized the UCSF-HSEC. A secondary aim was to elucidate the role that student-run clinics can play in connecting the homeless population to healthcare and community services.

\section{Methods}

A retrospective cohort study was performed examining patients who received care at the UCSF-HSEC. All patients seen at the monthly UCSF-HSEC from August 2017 to December 2019 were included. This study was approved by the Institutional Review Board at the University of California San Francisco. This study complied with the Health Insurance Portability and Accountability Act and followed the tenets of the Declaration of Helsinki.

At each monthly clinic, UCSF medical student volunteers canvassed all floors of the homeless shelter to offer comprehensive eye exams to the residents. All exams were conducted a dedicated clinic room in the homeless shelter. Patient rooms were partitioned off with portable room dividers and curtains for privacy. For the consenting participants, medical students obtained demographic information such as age, gender, race, ethnicity, birthplace, and education level along with a focused medical and ocular history. With regard to medical history, patients were only asked if they had a history of HTN, DM, or HIV/AIDS. A comprehensive ocular exam was then performed primarily by a senior resident with faculty attending oversight that comprised of visual acuity (near and far with a Snellen chart), extraocular movements, confrontational visual field, pupillary responses, intraocular pressure (Ton-Pen), autorefraction (Retinomax), and fundoscopy with an indirect ophthalmoscope. UCSF ophthalmology residents and attendings allowed medical students to practice their ocular examination skills. Students were provided with the opportunity to make the initial assessment and residents and faculty ophthalmologist worked with medical students to revise and refine their assessments and plans to assign The International Classification of Diseases, 9th Revision (ICD-9) and ICD-10. If indicated, a patient received health coaching by a medical student on their diagnosis along with smoking cessation counseling.

Visual impairment was defined as visual acuity of less than or equal to $20 / 40$ but better than 20/200 in the better seeing eye, while legal blindness was defined as visual acuity of less than or equal to $20 / 200$ in the better seeing eye. ${ }^{15}$ The visual acuity recorded was the corrected visual acuity when the patient had eyeglasses or contacts, or the uncorrected visual acuity when the patient did not have correction.

Patients were diagnosed as glaucoma suspects if the patient had at least one of the following findings: intraocular pressure was greater than $21 \mathrm{~mm} \mathrm{Hg}$, evidence of optic nerve involvement, or a visual field abnormality. ${ }^{16} \mathrm{~A}$ patient was diagnosed with glaucoma in our records if they endorsed receiving a prior diagnosis of glaucoma by an eye provider that was verified with information in their medical record at Zuckerberg San Francisco General Hospital (ZSFG).

Patients in need of follow-up care were given prearranged appointments at ZSFG and community partner, Project Homeless Connect who provided patients with free eyeglasses if indicated.

Data obtained from direct patient interviews, physical exam findings, and ICD-9/10 codes were all recorded and stored in a secure database.

Patient demographic characteristics and common comorbidities such as HTN and diabetes were assessed. The prevalence of common diagnoses was stratified by race and ethnicity.

\section{Results}

\section{Patient Demographics}

A total of 123 patients were evaluated at the UCSF-HSEC. The average age of the patients was 51 years with a range between 25 and 82 years. Of the 123 patients, $74 \%$ identified as male and $21 \%$ identified as female. The gender identity for the remaining $5 \%$ was unknown or the patients preferred not to disclose.

All of the patients stated that they were homeless. The average year of homelessness was 7 years with a range of 0 to 60 years.

An adapted version of the National Institute of Health classification for race and ethnicity was utilized. ${ }^{16}$ Thirty-three percent of patients were identified as Black/African-America, $27 \%$ identified as White, $11 \%$ preferred not to disclose, $8 \%$ identified with race/ethnicity categories other than the provided options, $7 \%$ identified as Hispanic/Latino, $6 \%$ identified as Asian, 6\% identified with multiple races and ethnicities, 2\% identified as American Indian or Alaska Native, and $2 \%$ identified as Native Hawaiian or Pacific Islander (- Fig. 1).

The highest education level achieved by participants breaks down as follows: $46 \%$ stated they had a high school diploma, $15 \%$ had completed junior high school, $13 \%$ had a bachelor's degree, $11 \%$ unknown, $9 \%$ completed vocational school, $5 \%$ had an associate's degree, $1 \%$ completed a master's degree, and $1 \%$ 


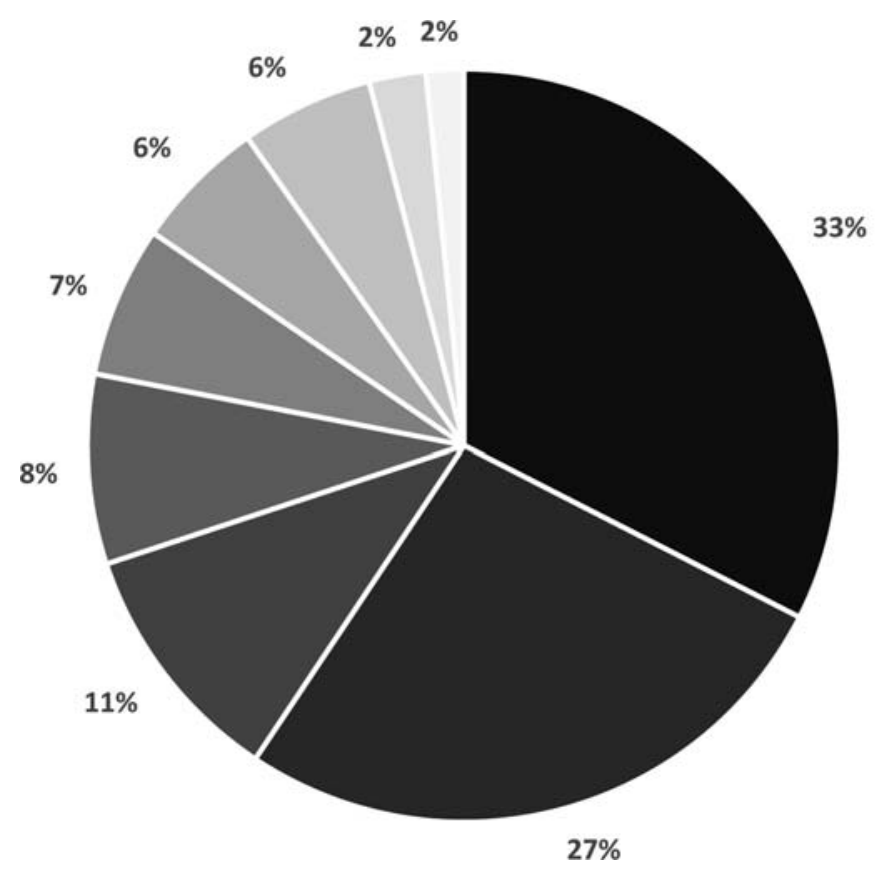

- Black or African American

- White

- Not to Disclosed / Unknown

- Other

- Hispanic

asian

= Multiple Races / Ethnicities

American Indian or Alaska Native

Native Hawaiian or Pacific Islander

Fig. 1 Racial and ethnic composition of patients attending the free eye clinic from 2017 to 2019.

stated that they had not completed any formal schooling

(-Fig. 2).

Seventy percent of patients were unemployed, $14 \%$ did not disclose their employment status, $8 \%$ stated they were retired, $7 \%$ were currently employed, and $1 \%$ were on disability (-Fig. 3).

\section{Medical History}

Sixty-five percent of patients had HTN, 27\% had DM2, and $8 \%$ had HIV/AIDS (-Fig. 4). Additionally, out of the 98 (80\%) patients who knew their family history, $43 \%$ stated they had a family history of eye disease. The following rates of substance use were noted: $38 \%$ endorsed tobacco use, $26 \%$ endorsed marijuana, 21\% endorsed alcohol, and 15\% endorsed illicit drug use (-Fig. 5). Fifty percent of the patients did not have a primary care physician (PCP).

\section{Ophthalmologic Findings}

Uncorrected near visual acuity (UNVA was collected in 55\% of the study participants (68/123). Sixty-eight percent (46/68) of the patients were classified as visually impaired by having an UNVA of $20 / 40$ or less, and $9 \%$ (6/68) were classified as

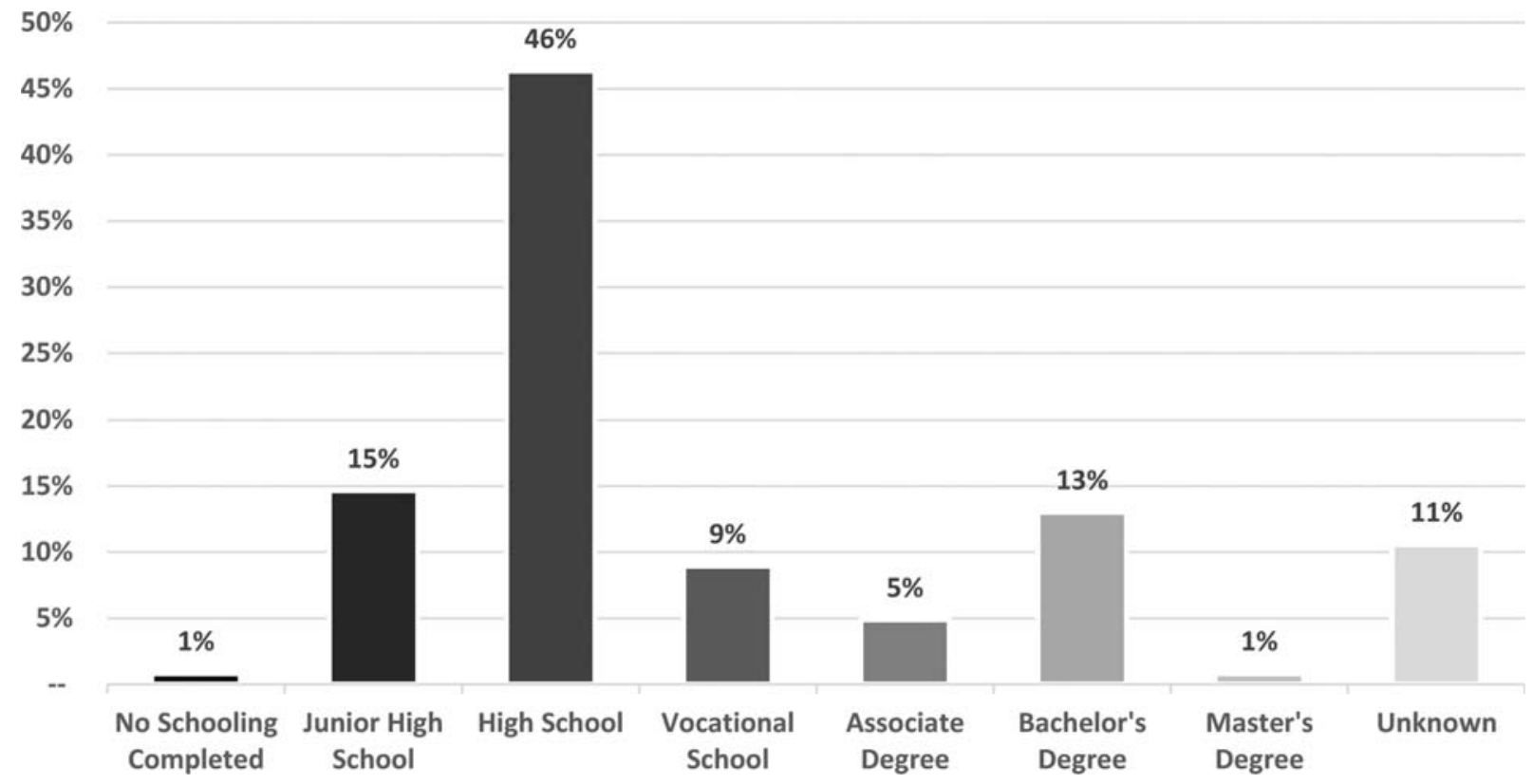

Fig. 2 Highest level of educational attainment of patients attending the free eye clinic from 2017 to 2019. 


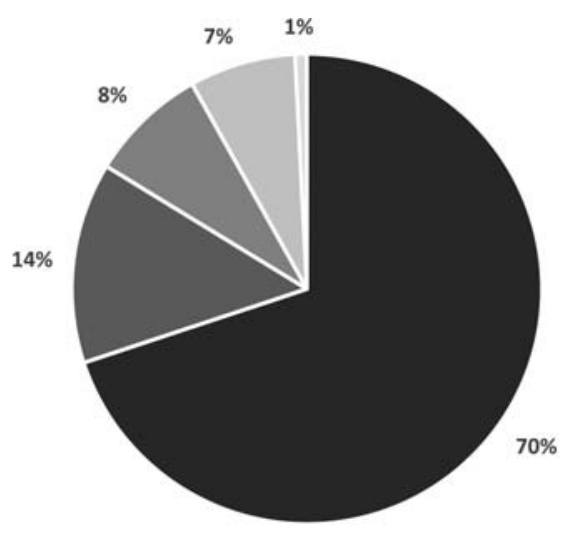

- Unemployed | Not Disclosed | Retired = Employed | Disability

Fig. 3 The employment status of patients attending the free eye clinic from 2017 to 2019. blind by having an UNVA of $20 / 200$ or less. The average uncorrected near logarithm of the minimum angle of resolution ( $\log$ MAR) was 0.43 with a standard deviation of 0.32 .

Corrected near visual acuity (CNVA) was collected in $62 \%$ (76/123) of the study participants. Forty percent (40/76) of the patients were classified as visually impaired by having a CNVA of $20 / 40$ or less, and $1.3 \%$ (6/76) were classified as blind by having a CNVA of 20/200 or less. The average corrected near logMAR was 0.21 with a standard deviation of 0.23 .

The most prevalent eye disease was refractive error at a prevalence rate of $70 \%$ with presbyopia and myopia accounting for 77 and $42 \%$ of the refractive error diagnoses, respectively.

A visually significant cataract was the second most prevalent pathology, which was diagnosed in $13 \%$ of patients. Diabetic retinopathy was categorized as proliferative and nonproliferative defined as the appreciation and absence of neovascularization on fundoscopy respectively. ${ }^{17}$ Diabetic retinopathy had a prevalence rate of $11 \%$ : $7 \%$ had nonproliferative diabetic retinopathy and $4 \%$ had proliferative retinopathy. Cranial nerve and

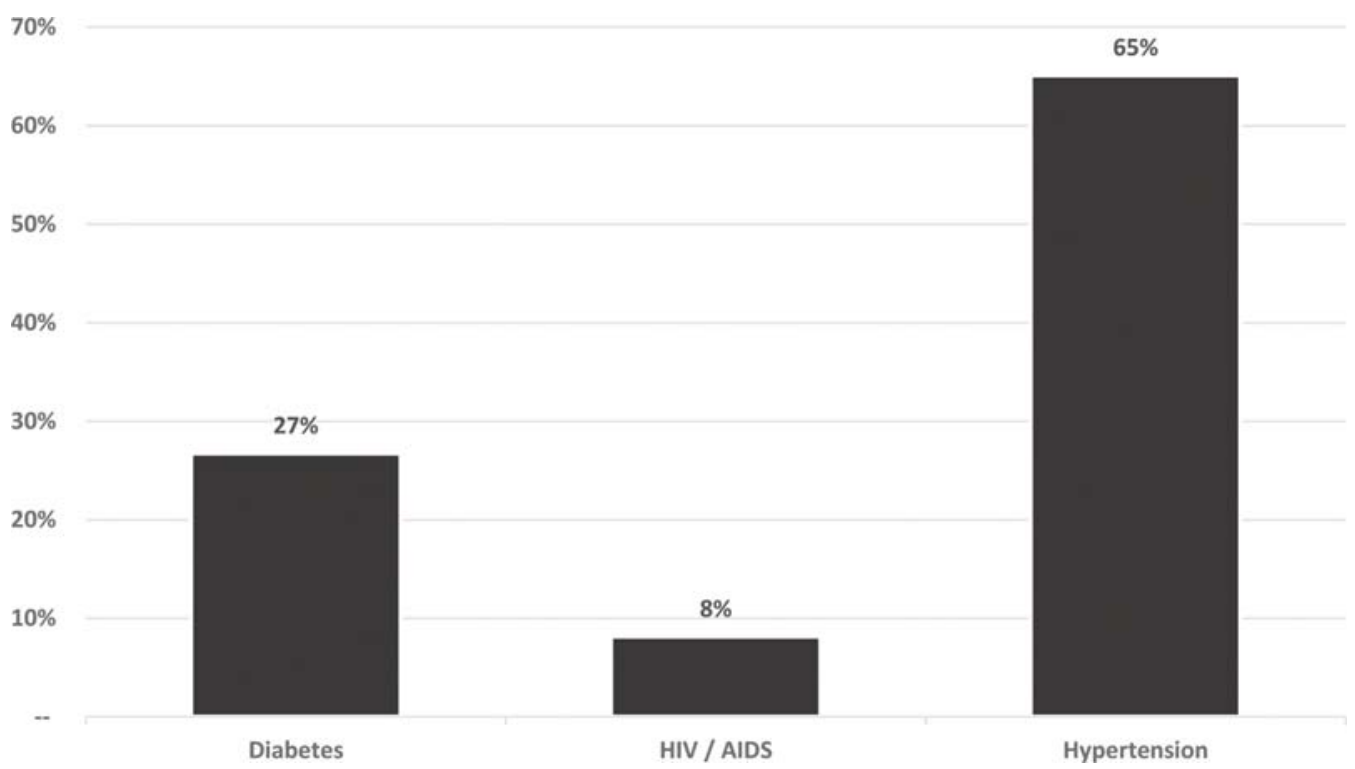

Fig. 4 The prevalence of comorbidities of patients attending the free eye clinic from 2017 to 2019.

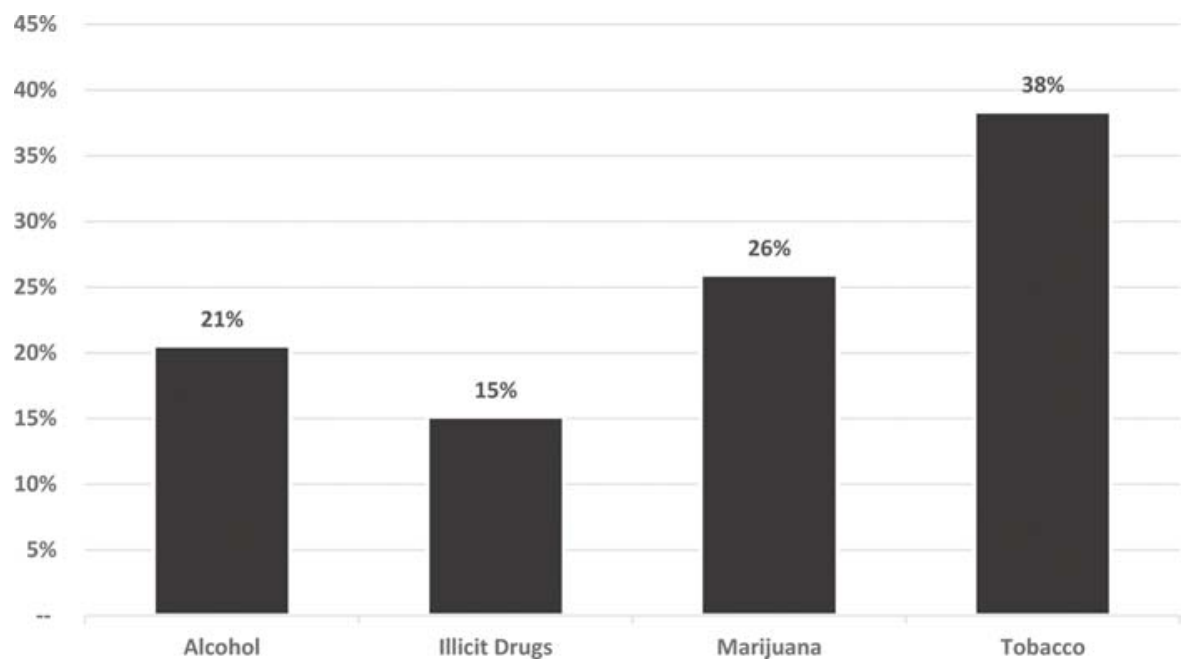

Fig. 5 The prevalence of substance use of patients attending the free eye clinic from 2017 to 2019. 
Table 1 Prevalence of ocular disease

\begin{tabular}{|l|l|}
\hline Diagnosis & Total \\
\hline Chorioretinal & \\
\hline AMD & 1 \\
\hline Chorioretinal scar & 1 \\
\hline Choroidal lesion suspicious & 1 \\
\hline Choroidal nevus & 1 \\
\hline Diabetic macular edema & 1 \\
\hline Hypertensive retinopathy & 1 \\
\hline Lattice & 1 \\
\hline Macular hole & 0 \\
\hline Macular scar & 1 \\
\hline Nonproliferative diabetic retinopathy & 9 \\
\hline Proliferative diabetic retinopathy & 4 \\
\hline Posterior vitreous detachment & 4 \\
\hline Retinal detachment & 1 \\
\hline Conjunctiva & \\
\hline Dry eye & 10 \\
\hline Pingueculitis & 1 \\
\hline Cornea & 2 \\
\hline Astigmatism & 16 \\
\hline Epithelial basement membrane dystrophy & 1 \\
\hline Epitheliopathy & 12 \\
\hline Cranial nerve & 2 \\
\hline Afferent pupillary defect & \\
\hline Bilateral hemianopsia & 8 \\
\hline Diplopia & 1 \\
\hline Duane's syndrome & 1 \\
\hline Ptosis & 1 \\
\hline Refractive amblyopia & 25 \\
\hline Strabismus & \\
\hline Visual field defects & 13 \\
\hline Eyelid & \\
\hline Blepharitis & \\
\hline Suspicious cancer of eyelids & 13 \\
\hline Glaucoma suspect/Glaucoma & \\
\hline Globe & 1 \\
\hline Eye trauma & 1 \\
\hline Iris coloboma & 1 \\
\hline Lacrimal & 1 \\
\hline Epiphora & 1 \\
\hline Lens & 1 \\
\hline Aphakia & 1 \\
\hline Cataracts & 1 \\
\hline
\end{tabular}

corneal lesions were appreciated in 17 and $11 \%$ of the patients, respectively, while $8 \%$ of patients were found to have normal ocular exams (-Table 1).

Glaucoma had a prevalence rate of $20 \%$. When stratifying the prevalence of glaucoma by race/ethnicity, Black/AfricanAmericans had a prevalence rate of $30 \%$, Asians had a prevalence rate of $29 \%$, Hispanic/Latinos had a prevalence rate of $25 \%$, and White/Caucasians had a prevalence rate of $18 \%$ (-Fig. 6).

\section{Discussion}

The ultimate purpose of this study was to assess the patient characteristics and prevalence of ophthalmologic diseases of patients receiving care at the institution-sponsored student operated eye clinic during a 2-year period. The main findings of this retrospective cohort study demonstrate that there is a higher prevalence of eye disease among the homeless population of San Francisco as compared with the overall prevalence rates of ophthalmologic disease nationally. ${ }^{18}$ Additionally, the high prevalence rates of comorbidities such as HTN, DM2, and HIV/AIDS reinforce the findings of prior studies that identified higher burdens of disease among the homeless. ${ }^{19}$ When analyzing the social determinants of health, participants in this study had lower levels of educational attainment and higher unemployment rates compared with both San Francisco and national averages. ${ }^{20,21}$

This study reiterates the presence of healthcare disparities since certain minorities were overrepresented in this study when compared with the prevalence of these ethnic and racial categories outlined in the records of San Francisco County and the national census. For example, the racial and ethnic composition of San Francisco is the following: 40\% White/Caucasian, $34 \%$ Asian, 15\% Hispanic, and 5\% Black/African-American. ${ }^{22}$ At the national level, the percentage composition of these populations is $60 \%$ White/Caucasian, $18 \%$ Hispanic/Latino, $13 \%$ Black/African-American, 13\% Asian, and 1\% American Indian and Alaska Native, and $0.2 \%$ Native Hawaiian or other Pacific Islander. ${ }^{23}$ In this study, the percentage of Black/African-American patients is 33\% that is seven times the average for San Francisco and three times the national average. Prior studies have demonstrated that Black/African-Americans and Asians have a higher susceptibility of developing glaucoma ${ }^{24,25}$ and the results in this study have reconfirmed these trends. This 


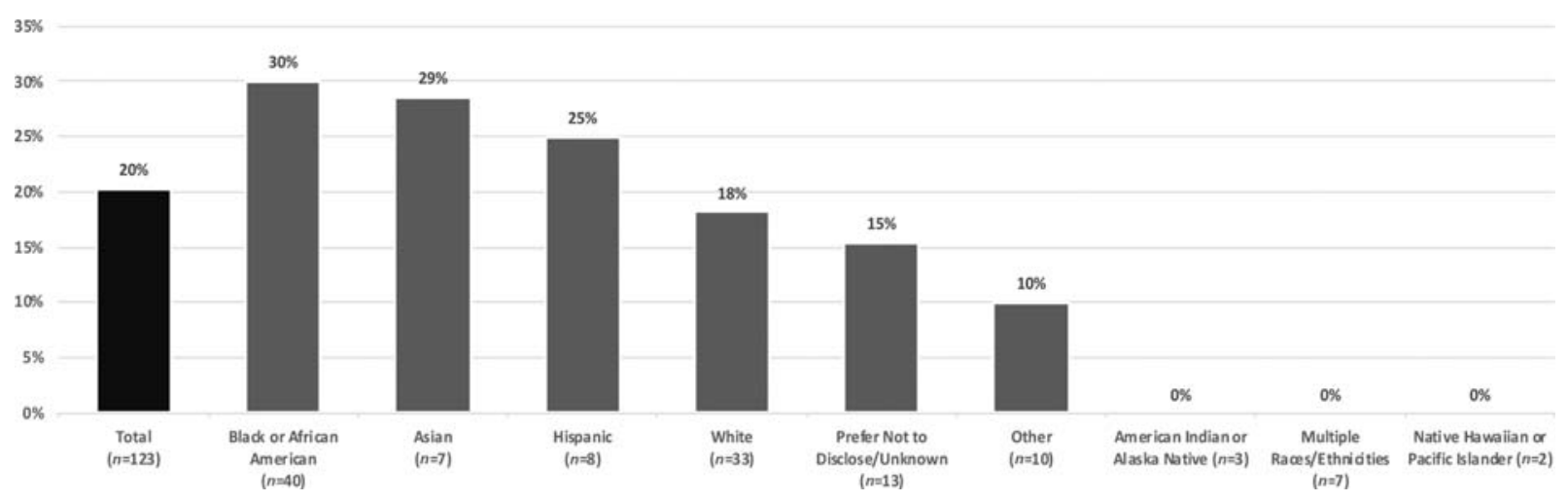

Fig. 6 The race and ethnicity of patients with glaucoma attending the free eye clinic from 2017 to 2019.

underserved population demonstrated a higher prevalence of ocular disease in aggregate when compared with the general population and thus highlights the major role that social determinants of health play in patient outcomes.

Limitations were identified in the collection of VA and thus the determination of visual impairment. UNVA was utilized for patients who never owned eyeglasses or contact lenses. UNVA was calculated instead of best corrected visual acuity for these patients and use of the former may have led to an overestimation of the prevalence of visual impairment among this population but it appeared to be a more accurate proxy of their visual acuity since they were less likely to obtain correction. To mitigate this concern, we have formed a partnership with a local organization Project Homeless Connect that provides free eyeglasses for our referred patients. Limitations were also centered around the patient selection process as patients with eye concerns were most likely to agree to receiving an eye examination. Patient selection was not randomized. Therefore, the context of this clinic may have led to an increased likelihood of attracting patients with preexisting eye diseases. Our study population may not be representative of the demographic and ocular pathology trends at the national level that limit the generalizability of these findings to general populations. However, the findings in this study are applicable to other micropopulations that consist of predominantly homeless and underserved populations since previous studies in this subset have discovered similar ophthalmologic trends. ${ }^{26,27}$ Lastly, as a retrospective study, it was hard to track patient outcomes over time and it was difficult to ascertain trends during this 2-year period.

Lastly, this study emphasizes the integral role student-run clinics play in providing healthcare to the community's most vulnerable who have a demonstrated need for ophthalmologic care. This study provided the opportunity to provide care for a growing population who often do not receive care within existing healthcare systems. Additionally, patients were connected to PCPs as 50\% of participants previously did not have a PCP. With the high prevalence of tobacco, alcohol, and marijuana, there are opportunities for health counseling and expansion of health education services that were provided by the premedical and medical students. Patients underwent health education sessions with hopes of understanding their illness and increased rate of adherence to medical care. In addition to providing increased opportunities to care for the community's most vulnerable, UCSF-HSEC provided increased exposure to the field of ophthalmology and increased opportunities for faculty and resident teaching and mentorship of students.

Future direction should assess the rates of follow-up to our partner primary care providers and ophthalmology providers at the local county hospital and community partner organization Project Homeless Connect. Additionally, the evaluation of the efficacy of our smoking cessation programs could be assessed. Further study could also investigate the impact of the clinic on medical student specialty choice and career focus.

\section{Funding}

This study was supported in part by an unrestricted grant from Research to Prevent Blindness, United States.

\section{Conflict of Interest}

None declared.

\section{Acknowledgments}

A special thank you to the California Endowment and Alcon Pharmaceuticals for their generous donations. A special thank you to all the premedical and medical student, resident, and faculty volunteers for their efforts in launching and running the clinic.

\section{References}

1 The Lancet. Health of the homeless Lancet 2014;384(9953):1478

2 State of Homelessness National Alliance to End Homelessness. https://endhomelessness.org/homelessness-in-america/homelessness-statistics/state-of-homelessness-report/. Accessed Sep 15,2020

3 Donaldson LP, Yentel D. Affordable housing and housing policy responses to homelessness. In: Larkin H, Aykanian A, Streeter CL, eds. Homelessness Prevention and Intervention in Social Work: Policies, Programs, and Practices. Springer International Publishing; 2019:103-122

4 Scott J, Gavin J, Egan AM, et al. The prevalence of diabetes, prediabetes and the metabolic syndrome in an Irish regional homeless population. QJM 2013;106(06):547-553

5 Oliveira Lde P, Pereira ML, Azevedo A, Lunet N. Risk factors for cardiovascular disease among the homeless and in the general population of the city of Porto, Portugal. Cad Saude Publica 2012; 28(08):1517-1529 
6 Lee TC, Hanlon JG, Ben-David J, et al. Risk factors for cardiovascular disease in homeless adults. Circulation 2005;111(20): 2629-2635

7 Pluck G, Lee K-H, Lauder HE, Fox JM, Spence SA, Parks RW. Time perspective, depression, and substance misuse among the homeless. J Psychol 2008;142(02):159-168

8 Kushel MB, Vittinghoff E, Haas JS. Factors associated with the health care utilization of homeless persons. JAMA 2001;285(02): 200-206

9 Noel CW, Srivastava R, Lo R, Berger A, Tehrani N, Lichter M. Unmet eye care needs among a homeless youth population. Can J Ophthalmol 2016;51(03):180-184

10 Noel CW, Fung H, Srivastava R, et al. Visual impairment and unmet eye care needs among homeless adults in a Canadian city. JAMA Ophthalmol 2015;133(04):455-460

11 Saeedi O, Ashraf H, Slade EP, et al. Trends in prevalence of diagnosed ocular disease and utilization of eye care services in American veterans. Am J Ophthalmol 2017;173:70-75

12 Davis JA, Tsui I, Gelberg L, Gabrielian S, Lee ML, Chang ET. Risk factors for diabetic retinopathy among homeless veterans. Psychol Serv 2017;14(02):221-228

13 Sawers N. The state of ocular health among London's homeless population. Eye (Lond) 2017;31(04):632-635

14 Macias EP, Lee DA, Oelrich FO. Refractive errors and visual acuity impairment among self-selected Hispanic, white, and black adults examined by the UCLA Mobile Eye Clinic. J Am Optom Assoc 1999; 70(11):724-734

15 Buch H, Vinding T, Nielsen NV. Prevalence and causes of visual impairment according to World Health Organization and United States criteria in an aged, urban Scandinavian population: the Copenhagen City Eye Study. Ophthalmology 2001;108(12): 2347-2357

16 The Glaucoma Suspect. Available at: https://www.aao.org/ bcscsnippetdetail.aspx?id=3267989c-d06f-4faf-8144-fc05139f80 cc. Accessed July 30, 2020
17 Diabetic Retinopathy for Medical Students Classification. Available at: https://eyerounds.org/tutorials/Diabetic-RetinopathyMed-Students/Classification.htm. Accessed Aug 1, 2020

18 NOT-OD-15-089: Racial and Ethnic Categories and Definitions for NIH Diversity Programs and for Other Reporting Purposes. Available at: https://grants.nih.gov/grants/guide/notice-files/not-od15-089.html. Accessed May 13, 2020

19 Varma R, Vajaranant TS, Burkemper B, et al. Visual impairment and blindness in adults in the United States: demographic and geographic variations from 2015 to 2050. JAMA Ophthalmol 2016;134(07):802-809

20 Beijer U, Andréasson S. Physical diseases among homeless people: gender differences and comparisons with the general population. Scand J Public Health 2009;37(01):93-100

21 U.S. Census Bureau QuickFacts United States. https://www.census, gov/quickfacts/fact/table/US/PST045219. Accessed April 3, 2020

22 Resnikoff S, Pascolini D, Etya'ale D, et al. Global data on visual impairment in the year 2002. Bull World Health Organ 2004;82 (11):844-851

23 Racette L, Liebmann JM, Girkin CAADAGES Group. , et al. African Descent and Glaucoma Evaluation Study (ADAGES): III. Ancestry differences in visual function in healthy eyes. Arch Ophthalmol 2010;128(05):551-559

24 Brown RT, Goodman L, Guzman D, Tieu L, Ponath C, Kushel MB. Pathways to homelessness among older homeless adults: results from the HOPE HOME Study. PLoS One 2016;11(05):e0155065. Doi: 10.1371 /journal.pone.0155065

25 San Francisco CA| Data USA. Available at: https://datausa.io/ profile/geo/san-francisco-ca. Accessed April 3, 2020

26 Mobile Eye Screening Hawaii Homeless.pdf | Powered by Box. Available at: https://ucsf.app.box.com/file/659044559864. Accessed May 10, 2020

27 East London's Homeless-a retrospective review.pdf | Powered by Box. Available at: https://ucsf.app.box.com/file/659053127527. Accessed May 10, 2020 